\title{
Epithelial-mesenchymal transition and estrogen receptor-dependent pathway are linked with ovarian cancer cell growth and migration caused by cigarette smoke extracts in human ovarian cancer cells
}

Geon-Tae Park, So-Ye Jeon and Kyung-Chul Choi Correspondence

Laboratory of Biochemistry and Immunology, College of Veterinary Medicine, Chungbuk National University, Cheongju, Chungbuk 361-763 Republic of Korea

\section{ABSTRACT}

Cigarette smoke contains over 60 well established carcinogens. And there are strong links between some of these carcinogens and various types of smoking-induced cancers. In this study we investigated whether cigarette smoke extracts (CSEs) affects the cell proliferation, migration and invasion activity by alteration of epithelial-mesenchymal transition (EMT) in BG-1 human ovarian cancer cells. We confirmed that CSEs increased the ovarian cancer cell viability in a dose-dependent manner. Also the protein expression of cyclin D1 and cyclin E1 was increased while p21 and p27 expression was decreased by treatment of all CSEs (3R4F and two-domestic cigarettes). Additionally the alteration of EMT markers such as $\mathbf{E}$-cadherian and $\mathrm{N}$-cadherin was examined. The expression of E-cadherin was reduced by the treatment of CSEs while its reverse transition marker $\mathrm{N}$-cadherin was increased. And EMT-associated transcriptional factors, Snail and Slug were up-regulated by CSEs treatment too. These results indicate that CSEs can increase the processing of EMT in BG-1 ovarian cancer cells. In general, the EMT process is associated with cancer cell migration, so we examined the migration activity through scratch assay and fibronectin coated trans-well invasion assay too. Our results demonstrated that CSEs increased cancer cell migratory propensity. These functional alterations were associated with changes in the metastasis-related genes. Upon CSEs stimulation, the expression of the MMP-9 and cathepsin D was increased too. From this study we confirmed that CSEs increased the growth of human ovarian cancer cells and the development of cancer metastasis. CSEs treatment leads to the process of EMT through the up- and down- regulation of a multiple cellular markers and signaling proteins. Based on the results of this study CSEs exposure might have a higher risk of ovarian cancer than nonsmokers. It is hoped that this study provide new insights for the prevention and cure of cigarette smoked induced cancers.

\section{RESULT}
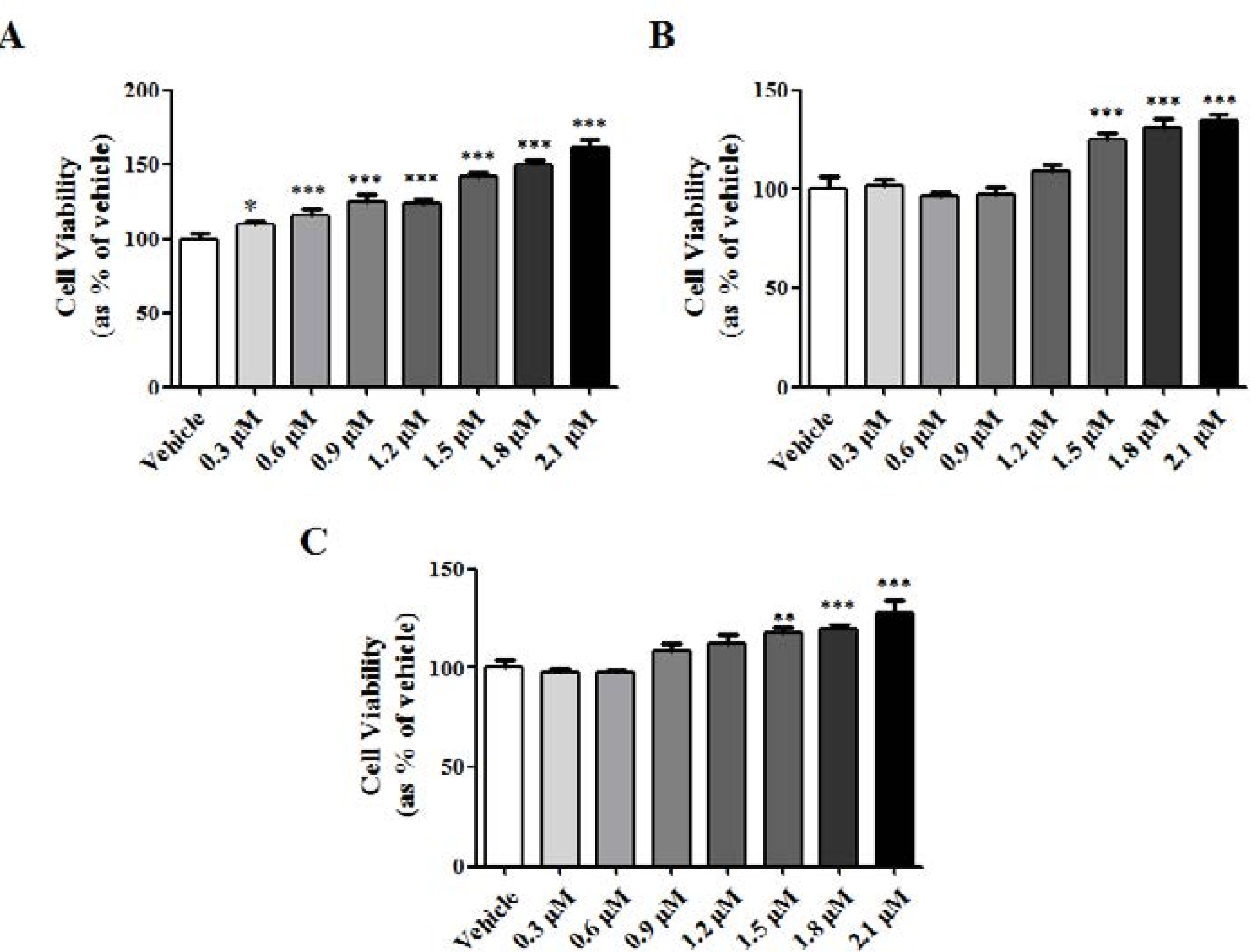

Figure 1. Effects of CSEs on cell proliferation in BG-1 cells. The effects of CSEs on BG-1 cell viability were measured at the various concentrations of three kinds of CSEs based on the concentration of nicotine. BG-1 cells were seeded at 5,000 cells per well in 96 well plates in phenol-red free DMEM containing 5\% CD-FBS. After 2 days, the medium was replaced by phenol red-free DMEM $(+5 \%$ CD-FBS) containing PBS and (A) $3 R 4 F$, (B) CSE1 and (C) CSE2 for 9 days. The cell viability was determined by MTT assay. Values are the means $\neq$ SD, * $P<0.05, * P<0.01$ and $* * * P<0.001$ compared control ).

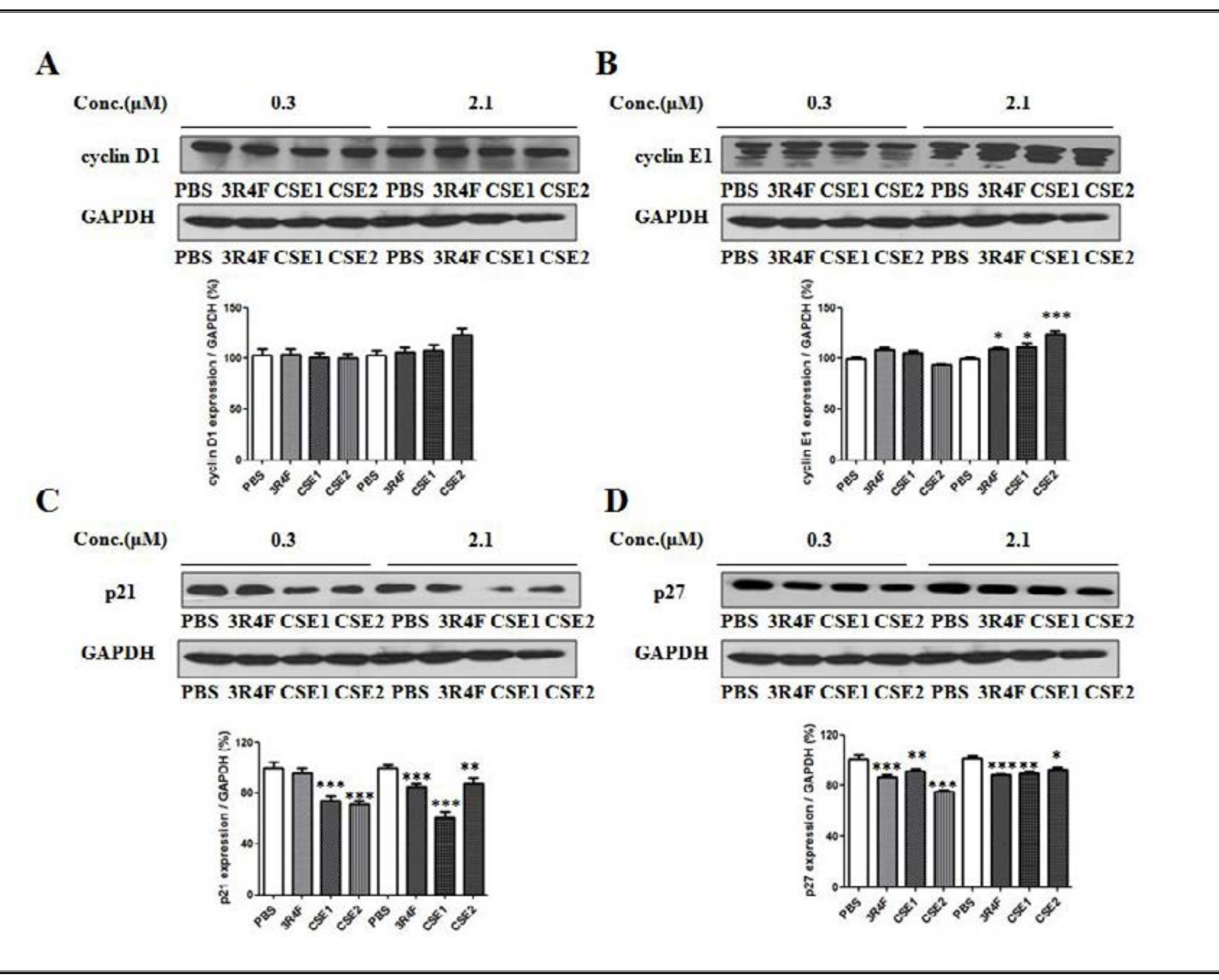

Figure 2. Effects of CSEs on the protein expressions of cell cycle related genes. Effects of CSEs on the expression of cell cycle related genes were examined by western blot in the translational levels. Data showed the alterations in (A) cyclin D1, (B) cyclin E1, (C) p21 and (D) p27 after 48 h of treatments in BG-1 cells. All experiments were done at least three times. Data were quantified using Gel Doc 2000. Each protein level was normalized against GAPDH expression. Values are the means \pm SD. ${ }^{*} P<0.05, * * P<0.01$ and $* * * P<0.001$ compared to vehicle (PBS).

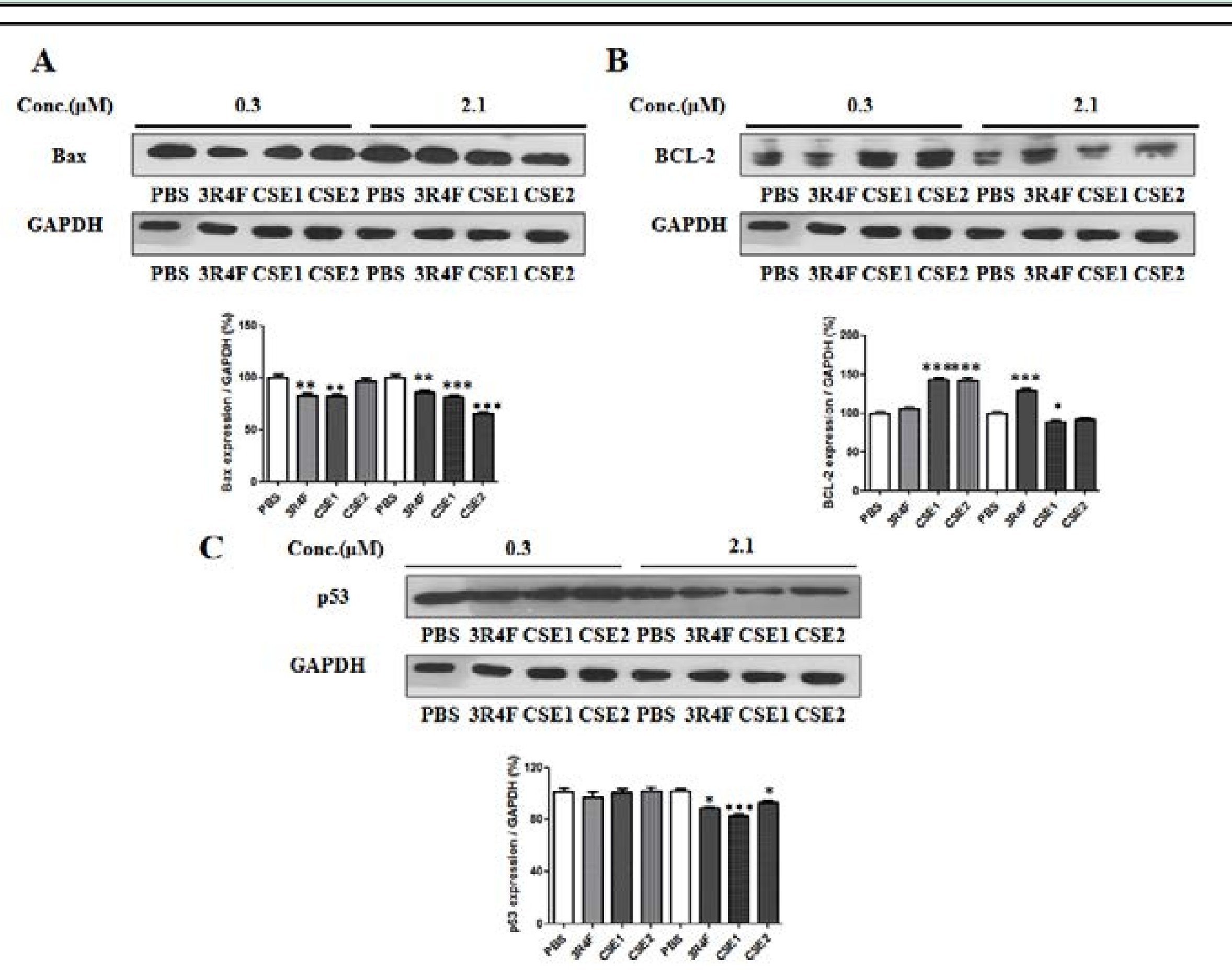

Figure 3. Alteration of apoptosis related gene expression by CSEs treatment in protein levels Effects of CSEs treatment on the expression of apoptosisrelated genes were investigated by western blot in the translational levels. Data showed the alterations in (A) Bax, (B) BCL-2, and (C) p53 after $48 \mathrm{~h}$ of treatments in BG-1 cells. Data were quantified using Gel Doc 2000. Each protein level was normalized against GAPDH expression. Values are the means \pm SD. ${ }^{*} P<0.05, * * P<0.01$ and $* * * P<0.001$ compared to vehicle (PBS).

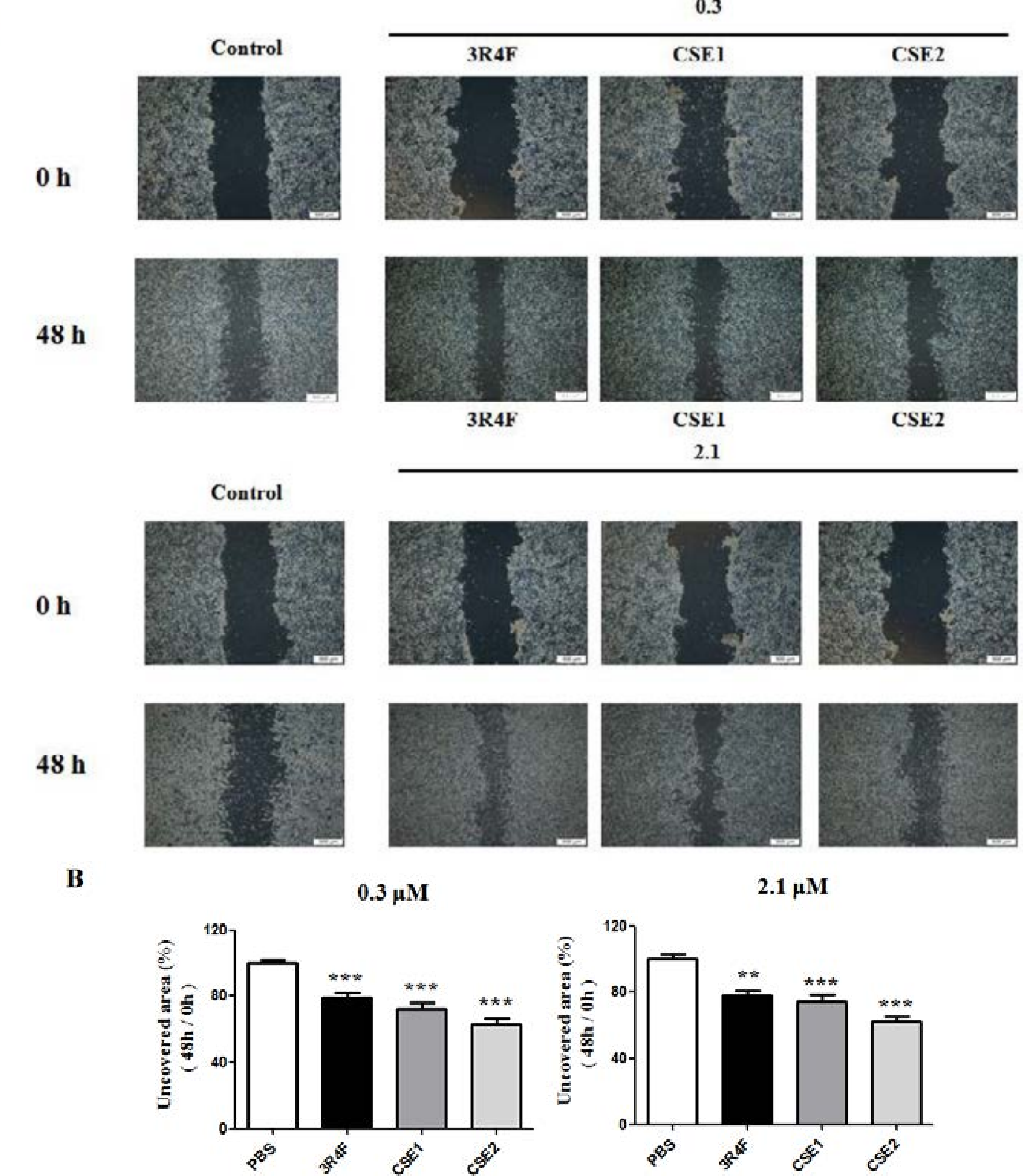

Figure 4. Altered migration activity of BG-1 cells by treatment with CSEs BG-1 cells were seeded and then incubated until $80 \%$ of confluen growth. (A) Altered cell migration by the treatment of CSEs was investigated using scratch assay. The protocol was described in Materials and Methods. Each image was observed by microscope under $4 \times$ magnifications. (B) Quantification of uncovered areas was conducted by using an eXcope Lite program. All experiments were done at least thre times. Values are the means \pm SD $* P<0.05, * * P<0.01$ and $* * * P<0.001$ compared to control (PBS).
comes. Values are the mean

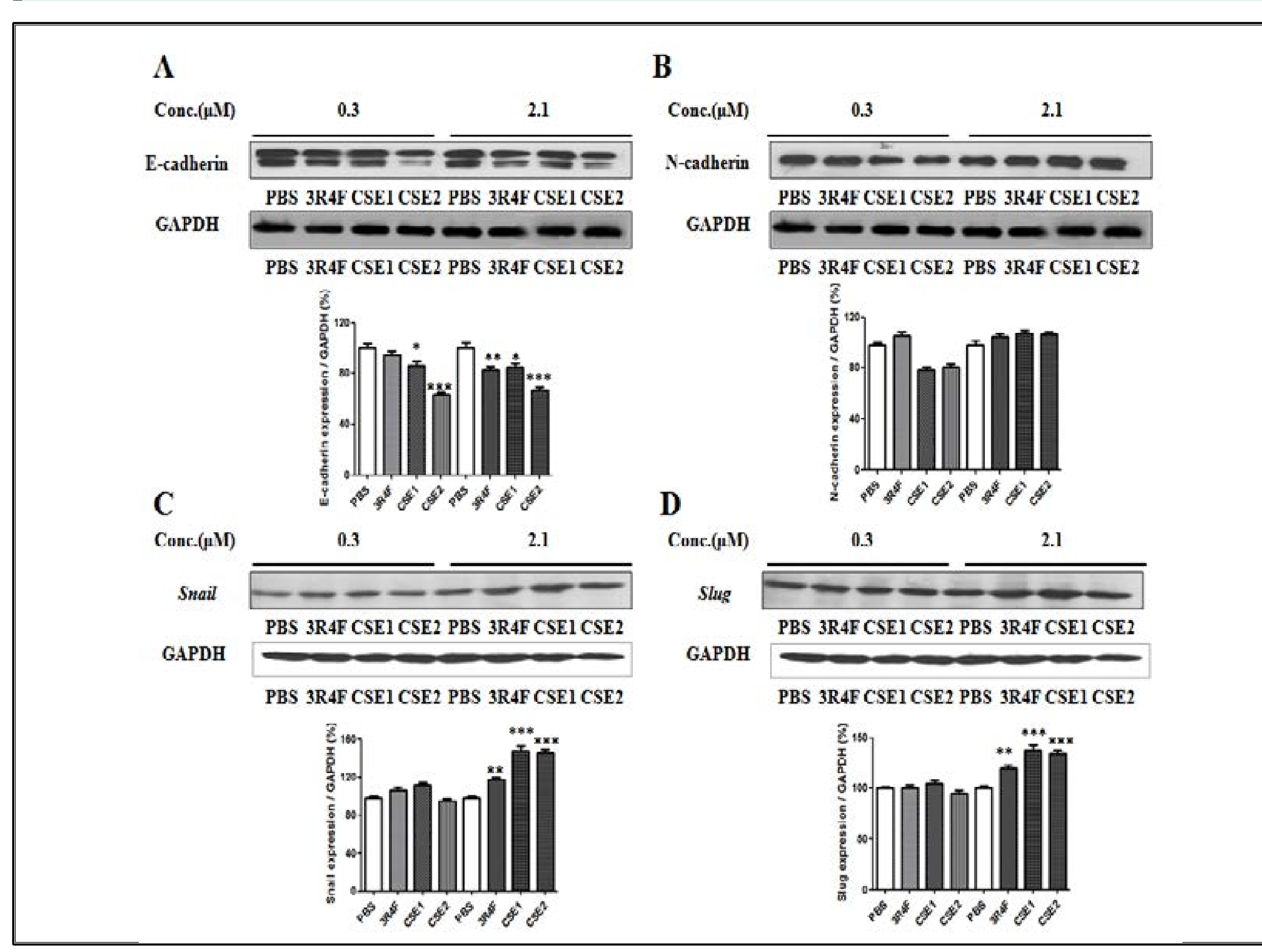

Figure 5. Alteration of EMT related gene expression by CSEs treatment in protein levels Effects of CSEs treatment on the expression of EMT-related genes were investigated by western blot in the translational levels. Data showed the alterations in (A) $\mathrm{E}$ cadherin, (B) N-cadherin, (C) Snail and (D) Slug after $48 \mathrm{~h}$ of treatments in BG-1 cells. Data were quantified using Gel Doc 2000. Each protein level was normalized against GAPDH expression.

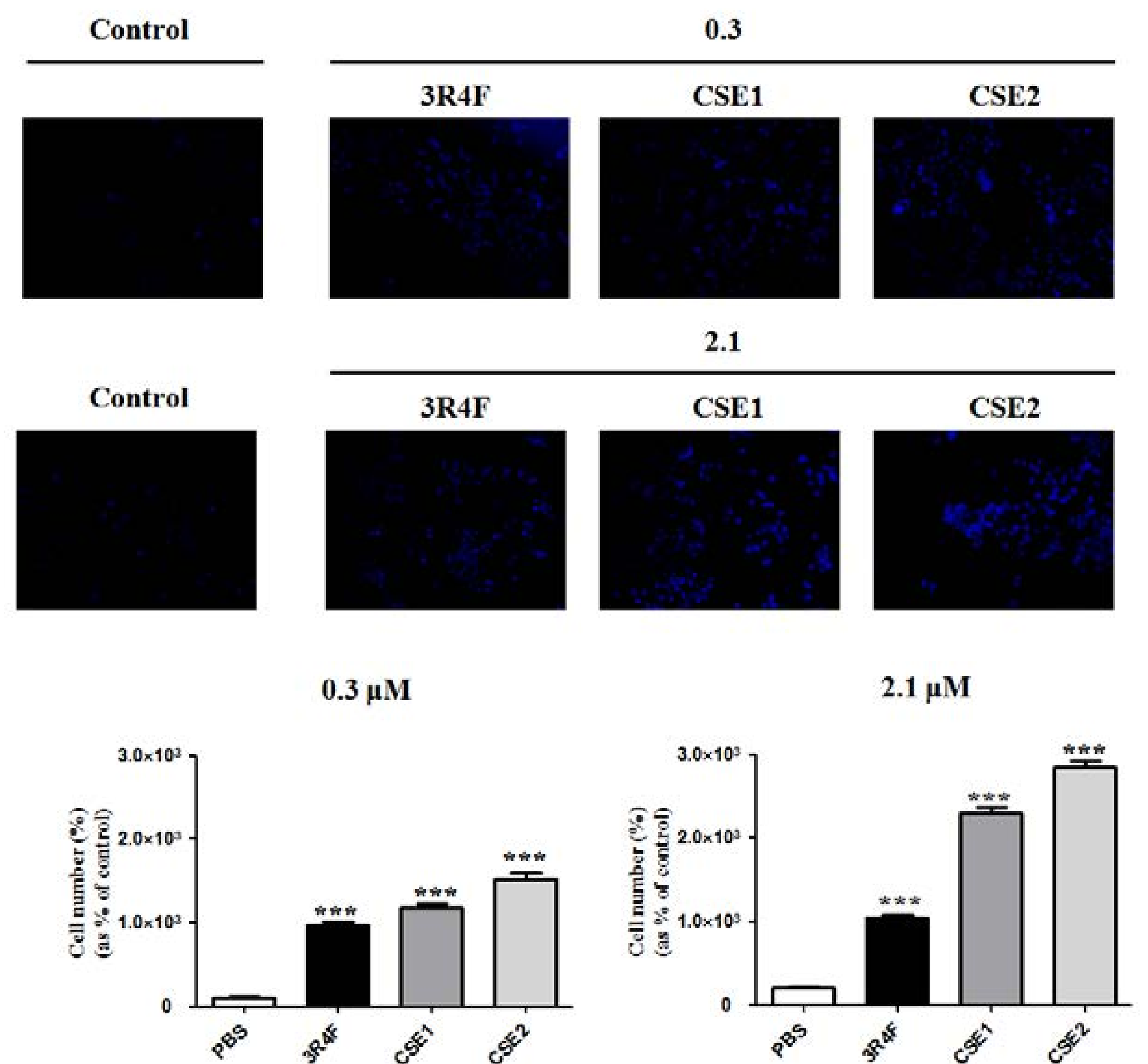

Figure 6. Altered cell invasive ability of BG-1 cells in the response of CSEs. Invasion assay was conducted to estimate BG-1 cells invasion in the presence of diverse treatments of CSEs. (A) Images captured by using microscope represent the lower chamber membrane of fibronectin-coated transwells after removing all non-invasive cells. (B) Invaded cells were quantified using the Image $\mathbf{J}$ software. To quantify cells that invaded from the upper chamber the cells were stained with DAPI.

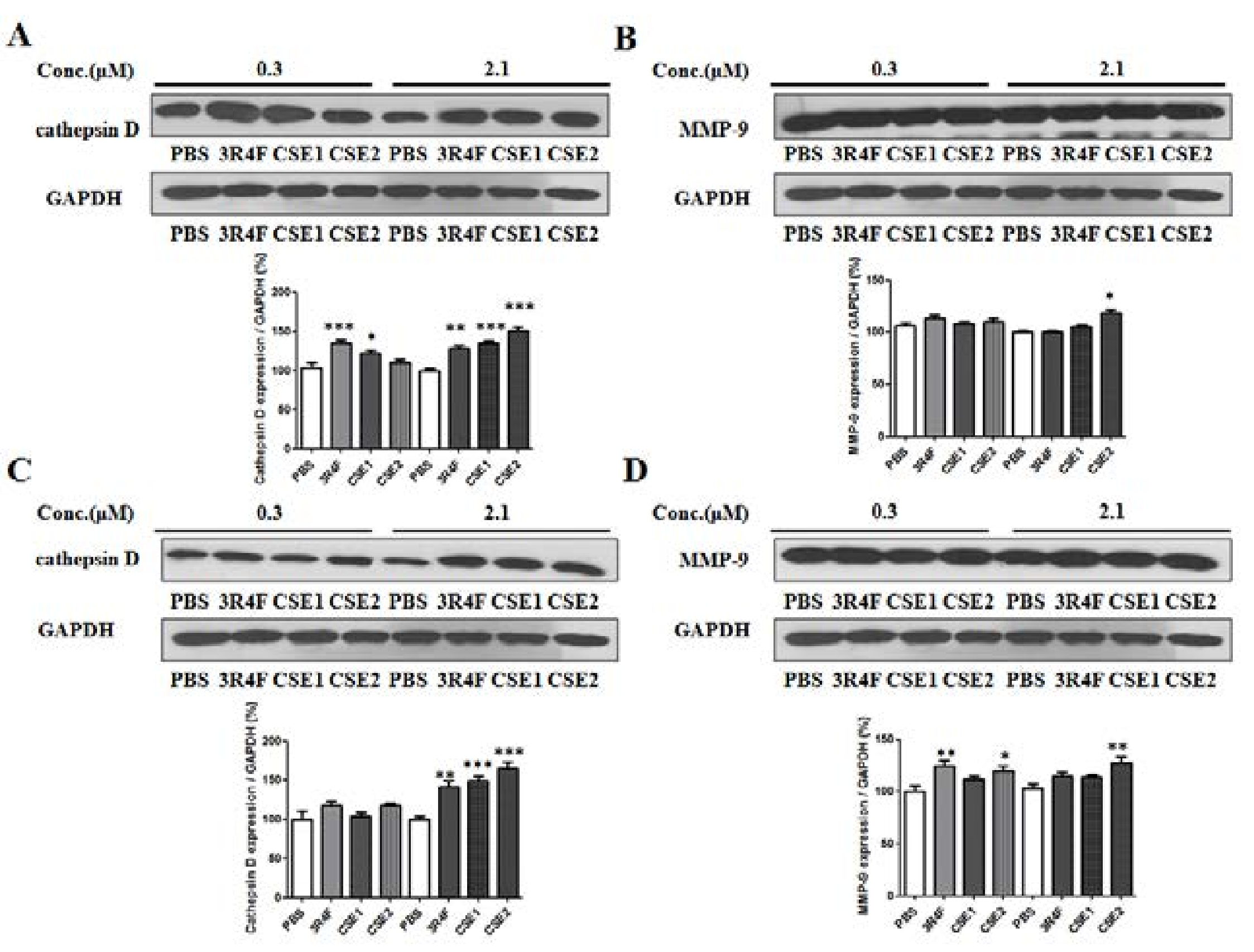

Figure 7. Effects of CSEs on the metastasis-related protein expression in BG-1 cells The effects of CSEs on expression of cathepsin D in BG-1 cells were investigated at each time point (24, 48 h) by western blot assay. Data showed the alterations in (A, C cathepsin D (B, D) MMP-9 after 24 and 48 h of treatments in BG1 cells. Data were quantified using Gel Doc 2000. Each protein level was normalized against GAPDH expression. Values are the means \pm SD. $* P<0.05, * * P<0.01$ and $* * * P<0.001$ compared to vehicle (PBS).

\section{CONCLUSION}

In conclusion, CSEs increased proliferation and metastatic potential of BG-1 human ovarian cancer cells by stimulating the growth, migration and invasion of BG1 cells. In parallel, CSEs affected the expression of cell cycle related genes in a way to promote cell cycle transition as well as inhibited the expression of proapoptotic genes, leading to a decreased apoptosis. Also CSEs treatment promoted the EMT process through the up- and down- regulation of a multiple cellular markers and signaling proteins. These functional alterations were associated with changes in the metastasis-related genes such as MMP-9 and cathepsin D. Based on the results, CSEs exposure might have a higher risk of ovarian cancer than nonsmokers. Also it is hoped that this study provide new insights for the prevention and cure of $C S$ induced cancer. 